
allemande

46-2 | 2014

Intellectuels et politique en Allemagne

\title{
Septembre 1944 : il faut évacuer le camp de Natzweiler
}

Robert Steegmann

\section{(2) OpenEdition}

Édition électronique

URL : https://journals.openedition.org/allemagne/1853

DOI : 10.4000/allemagne.1853

ISSN : 2605-7913

Éditeur

Société d'études allemandes

\section{Édition imprimée}

Date de publication : 30 décembre 2014

Pagination : 467-482

ISSN : 0035-0974

\section{Référence électronique}

Robert Steegmann, "Septembre 1944 : il faut évacuer le camp de Natzweiler », Revue d'Allemagne et des pays de langue allemande [En ligne], 46-2 | 2014, mis en ligne le 29 juillet 2019, consulté le 20 mai 2021. URL : http://journals.openedition.org/allemagne/1853 ; DOI : https://doi.org/10.4000/

allemagne.1853 


\section{Septembre 1944: il faut évacuer le camp de Natzweiler}

\section{- Robert Steegmann*}

Le $1^{\text {er }}$ septembre 1944, le commandant du camp de Natzweiler (KL-Natzweiler ${ }^{(1)}$ ), Fritz Hartjenstein, SS-Sturmbannführer ${ }^{(2)}$, transmet l'ordre reçu le jour même depuis les services centraux de l'IKL d'Oranienburg, d'évacuer le camp: "Conformément à l'appel téléphonique de l'Amstgruppe- $\mathrm{D}$, en date du $1^{\text {er }}$ septembre 1944, le KLNatzweiler doit être évacué ${ }^{(3)}$. Dès le lendemain, il commence à être mis à exécution. Un nouveau chapitre s'ouvre - dont tout porte à penser au départ qu'il ne saurait être définitif - dans l'histoire du système concentrationnaire nazi, mais aussi du Troisième Reich. «La Fin ${ }^{(4)}$ passe aussi par le KL-Natzweiler.

Ce camp, encore largement méconnu au regard des grandes structures du système concentrationnaire nazi dont certaines sont en place dès 1933, connaît alors un nouvel épisode d'une histoire qui est bien particulière et originale tout en s'inscrivant dans la " normalité " concentrationnaire. Il nous faudra brièvement la retracer, avant de retrouver la suite donnée à l'ordre reçu par Hartjenstein : car évacuer n'est ici pas libérer. Déployé de part et d'autre du Rhin par son réseau de camps annexes (kommandos), Natzweiler poursuit son existence, à compter de septembre 1944, sur la seule rive droite du fleuve. Il n'est plus dans son lieu d'origine et d'appellation, mais il continue de créer des nouvelles structures qui accueillent une population concentrationnaire

* Professeur de chaire supérieure et docteur en histoire, Strasbourg.

1 Nous utiliserons par la suite l'abréviation KL (Konzentrationslager) qui est d'usage dans les documents administratifs nazis, de préférence à celle de « $\mathrm{KZ}$ » usitée par l'historiographie allemande.

2 Pour une biographie, voir Robert Steegmann, Struthof. Le KL-Natzweiler et ses kommandos. Une nébuleuse concentrationnaire des deux côtés du Rhin, 1941-1945, Strasbourg, La Nuée-Bleue, 2005, p. 328-330.

3 Bundesarchiv Ludwigsburg, (BAL) Metz Ordner 7, P.V. Hartjenstein, $1^{\text {er }}$ septembre 1944. Traduction intégrale in: R. SteEgmanN, Struthof (note 2), p. 159-160.

4 Nous reprenons ici le titre éponyme de l'ouvrage de Ian Kershaw, Paris, Le Seuil, 2012. 
toujours plus nombreuse. Il mène son activité jusqu'au terme de l'histoire du Troisième Reich et, ici aussi, « le drame allait se jouer jusqu'au dernier acte » ${ }^{(5)}$.

\section{Un camp tardif dans un système déjà bien en place}

L'histoire du système concentrationnaire nazi débute bien avant le 21 mai $1941^{(6)}$. Ce jour, 150 détenus arrivent à la gare de Rothau sise cinquante kilomètres à l'ouest de Strasbourg dans la vallée de la Bruche. Deux jours plus tard, ils sont rejoints par 150 autres, eux aussi du KL-Sachsenhausen. Ils sont les premiers détenus du camp de Natzweiler.

Le camp s'inscrit dans une histoire commencée bien en amont à partir du 30 janvier 1933. L'ouverture du premier camp, le 3 mars 1933, sur l'aérodrome de Nohra, dans une Thuringe aux mains des nazis depuis 1932, s'inscrit dans le contexte « d'improvisation rageuse " ${ }^{(7)}$ qui est celui de 1933 . Sa brève existence - dix semaines - et sa faible capacité - moins de 200 détenus - n'en marquent pas moins l'inflexion donnée par les nouvelles règles juridiques.

Dès le lendemain de l'incendie du Reichstag, Hindenburg ratifie le décret-loi sur la "protection du peuple et de l'État " (Verordnung des Reichspräsidenten zum Schutz von Volk und Staat) qui suspend tous les droits fondamentaux qui peuvent encore être garantis par la constitution de Weimar et déjà bien rognés par une série de décrets dont celui du 4 février qui prolongeait la garde à vue de vingt-quatre heures à trois mois. Le nouveau décret-loi renforce la procédure de la Schutzhaft (« internement administratif préventif ») et supprime l'inviolabilité de la liberté individuelle. Il est en place jusqu'en 1945 et constitue l'outil principal de l'internement, arme suprême ne nécessitant aucune justification légale. Aucune perspective de jugement n’est prévue pour tous ceux qui, communistes, syndicalistes, sociaux-démocrates, intellectuels et autres, sont déclarés adversaires du nouveau régime. Procédure et durée de l'internement sont aux mains de la seule police des Länder.

Avec les premiers camps, improvisés et toujours brutaux (les " camps sauvages »), placés sous la coupe des SA ou des SS, se développe ce qui allait rapidement constituer un véritable système concentrationnaire rapidement contrôlé par Himmler et ses services $^{(8)}$.

Chef de la police de Munich (9 mars 1933), Himmler ${ }^{(9)}$ procède à l'ouverture du KL de Dachau, le 21 mars 1933, et nomme, le 26 juin, Theodor Eicke ${ }^{(10)}$ à sa tête qui en

5 I. Kershaw, La Fin, ibid., p. 382.

6 La synthèse la plus complète et la plus récente est: Wolfgang Benz, Barbara Distel (dir.), Der Ort des Terrors. Geschichte der nationalsozialistischen Konzentrationslager, 9 vol., Munich, C.H. Beck, 2005-2009.

7 Martin Broszat, "Nationalsozialistische Konzentrationslager 1933-1945 », in: H. BuCHheim, M. Broszat, H.A. Jacobsen (dir.), Anatomie des SS-Staates (1944), Munich, DTV, 1967, p. 323.

8 Pour l'histoire générale du système concentrationnaire, nous renvoyons principalement à M. BRosZAT, «Nationalsozialistische Konzentrationslager» (note 7); Karl ORTH, Die Konzentrationslager-SS. Sozialstrukturelle Analysen und biographische Studien, Göttingen, Wallstein, 2000; Benz/DisTeL (dir.), Der Ort des Terrors (note 6), vol. 1 : Die Organisation des Terrors, Munich, C.H. Beck, 2005.

9 Sur Himmler, voir la plus récente synthèse: Peter Longereich, Himmler, Paris, Éditions Eloïse d'Ormesson, 2010.

10 Sur Eicke et son passage à Dachau, voir P. Longereich, Himmler, ibid., p. 155 sq. 
fait un camp-modèle pour tous les suivants. Son règlement sert de base aux autres, et Dachau devient l'étape quasi obligée de la formation des cadres SS qui sont par la suite nommés ailleurs. Le décret du 10 décembre 1934 crée l’IKL, Inspection générale des camps dirigée par Eicke.

L’ouverture du KL de Sachsenhausen (été 1936) pose les jalons structurants de ce système, avec son administration et ses gardiens pris dans les SS-Totenkopfverbände. Le 27 septembre 1939 naît le RSHA (Reichssicherheitshauptamt - Office central de la sécurité du Reich) - confié à Heydrich. La police politique et criminelle, sous la direction centralisée de Himmler et de Heydrich, a désormais la haute main sur la détention. La Schutzhaft peut devenir «l'arme la plus tranchante du régime » (Heydrich) et, avec l'IKL désormais installée dans ses propres locaux à Oranienburg à proximité de Sachsenhausen, les hommes et les structures sont en place pour n'avoir qu'un seul objectif: être efficaces.

Le système concentrationnaire est fixé à partir de 1936. Ne reste plus qu'à le développer pour donner un cadre à son objectif. Des anciens KL restent Lichtenburg (devenu entre-temps camp de femmes) et Dachau, transformé et étendu en 1937, alors qu'ouvre le KL de Buchenwald (15 juillet). Flossenbürg (3 mai 1938), Mauthausen en Autriche rattachée (8 août 1938) et Ravensbrück (15 mai 1939) suivent.

De nouveaux camps appellent de nouveaux détenus. La détention s'élargit. Aux adversaires politiques de la première heure (Schutzhäftlinge) distingués par le port d'un triangle rouge, se rajoutent ceux pour lesquels il suffit de légaliser l'internement, d'élargir la taxinomie concentrationnaire et de les rendre distinguables par la couleur de leur triangle ${ }^{(11)}$. Les « verts" sont les Befristete Vorbeugungshäftlinge $(\mathrm{BV})^{(12)}$, puisés dans le monde de prisons parmi les criminels jugés dangereux et récidivistes; les «violets ", les témoins de Jéhovah (Bibelforscher), internés dès 1933. Les premiers servent le plus souvent d'auxiliaires pour l'auto-administration des camps, les seconds sont souvent utilisés pour des travaux internes. Se rajoutent les populations estimées racialement dangereuses par la loi « sur la prévention des tares héréditaires ». Avec l'opération Arbeitscheu Reich de 1938 ce sont les « rétifs au travail » qui mettent « en danger la société par [leur] comportement asocial » qui sont massivement internés. Formidable invention sémantique que celle de l'« asocial » qui permet de mener en camp et d'exclure de la société toute personne estimée " déviante ». Ils portent le triangle noir. Les homosexuels, accusés en vertu du paragraphe 175 de la loi allemande, ont un triangle rose. Les effectifs passent de 4761 en novembre 1936 à près de 24000 en octobre 1938 et les non politiques sont alors majoritaires. Après la nuit de Cristal (9-10 novembre 1938) près de 30000 Juifs sont internés dans différents camps.

Les années 1937-1938 voient l'augmentation du nombre des détenus, mais aussi une première mutation de leur emploi. La SS développe son réseau d'entreprises. Après l'acquisition de la manufacture de porcelaine d'Allach ${ }^{(13)}$ vient la Deustche Erd- und

11 Voir Robert Steegmann, Le camp de Natzweiler-Struthof, Paris, Le Seuil, 2009, p. 36 sq., pour la chronologie de cette évolution et ses fondements « légaux».

12 La langue des camps les nomme également Berufsverbrecher (criminels professionnels).

13 Hermann Kaienburg, Die Wirtschaft des SS, Berlin, Metropol, 2003; Albert Knoll, « Die Porzellanmanufaktur München-Allach », Dachauer Hefte, nº 15 (1999), p. 116-133. 
Steinwerke GmbH (DEST: "Entreprise des terres et pierres allemandes »). Nous la retrouverons à Natzweiler. D’autres suivent ${ }^{(14)}$ pour former le complexe économique de la SS. L'activité économique et les gains y afférents poussent à l'augmentation du nombre des détenus et à la création, en 1939, d'un Office central d'administration et d'économie (Hauptamt Verwaltung und Wirtschaft, HVW) placé sous la direction d'Oswald Pohl. À la fonction répressive et politique, les KL rajoutent ainsi une fonction économique.

La guerre oblige à une nouvelle mutation du système. À l'extension des territoires conquis répond la création de nouvelles structures: Auschwitz et Neuengamme en 1940, Groß Rosen et Natzweiler, décidés en 1940, ouvrent en 1941. Suivent encore Niederhagen (septembre 1941-avril 1943), Struthof (janvier 1942) et le SS-Sonderlager de Hinzert qui, bien que dépendant de l'IKL désormais dirigé depuis mai 1940 par Glücks, n'a pas encore le statut de KL. Les effectifs enflent, car à l'ennemi de l'intérieur s'ajoute désormais celui de l'extérieur - 21400 en août 1939, 80000 en mars 1942 -, et aux besoins politiques, l'internement répond aussi à un besoin économique. De nouvelles catégories apparaissent: les KGF (Kriegsgefangene), les NN (Nacht und Nebel). Bientôt les détenus allemands ne sont plus majoritaires et, ailleurs, se développe la politique systématique d'extermination.

La création du SS-WVHA (Wirtschaftsverwaltungshauptamt) en février 1942 est une autre étape fondamentale. Dirigé par Pohl, il intègre l'IKL en mars. Pohl a donc ainsi la haute main sur la mobilisation des détenus pour le travail donnant à Himmler un formidable réservoir de main-d'œuvre servile qu'il ne tarde pas à mettre à profit pour se rendre indispensable et pour accumuler les profits. Les conflits internes entre dirigeants sont monnaie courante dans le Reich et les acteurs sont puissants. Dans le cadre concentrationnaire, l'opposition d'intérêts entre Himmler, Goering - à la fois ministre et directeur des Hermann Goering Werke - et Alfred Speer, le favori de Hitler et ministre de l'Armement et des Munitions depuis 1942, ne cesse de grandir. Si les militaires répugnent à laisser l'industrie de guerre passer aux mains de la SS, et si les industriels ne consentent pas à voir l'ouverture de centres de production au sein des KL, c'est à Hitler de trancher tout en connaissant l'atout maître du seul Himmler: une main-d'œuvre qui peut être renouvelée et augmentée sans fin dans toute l'Europe. Contre une compensation - $5 \%$ des armements produits par les KL vont à la Waffen-SS - Himmler accepte de casser l'unité des camps. Les entreprises ne s'installeront pas dans les camps mais les détenus viendront vers elles. Nécessité et urgence font alors loi, mais la querelle des ego demeure, ignorant les principales victimes que sont les détenus qui, plus que jamais, ont " une âme d'esclave » ${ }^{(15)}$. Le travail ne les rendra pas libres.

Les camps annexes (kommandos) sont nés. Ils se rajoutent aux camps-souche (Hauptlager ou Stammlager) dont ils dépendent administrativement et peuvent même créer à leur tour un réseau de sous-camps (Nebenkommandos). La terreur a son cadre.

14 On peut citer la Deutsche Versuchanstalt für Ernährung und Verpflegung, la Deutsche Ausrüstungswerke (DAW) à Dachau, la Texled (Textil- und Lederverwertung GmbH) à Ravensbrück.

15 Doc. Nuremberg, PS-1992a. Discours de Himmler sur la nature et la tâche de la police, 15-23 novembre 1937 . 
Il faut produire, mais aussi faire du profit et marquer sa sphère d'influence de manière prééminente. Une véritable toile de camps annexes se tisse dès lors à travers tout le Reich, au plus près des usines, mais également des habitants. Les camps sont désormais « devant la porte " (vor der Haustür $)^{(16)}$, et c'est toute l'Europe qui y participe par le travail forcé et la répression qui s’accroît.

Alors que plus à l'écart de la population allemande s'ouvrent et fonctionnent les camps d'extermination (Belzec, Sobibor, Treblinka, Chelmno, Birkenau), alors que continuent de se remplir - avant d'être vidés - les ghettos, en Allemagne, dès la fin de 1942, 82 camps annexes sont déjà en place. Leur croissance est inéluctable dans un Reich désormais embourbé dans une guerre totale avec l'URSS, qui n'en termine pas et qu'il ne peut pas perdre. Les structures sont de plus en plus éclatées en réponse aux besoins qui se font plus pressants et nombreux. Les bombardements alliés qui s'intensifient sur l'Allemagne dès 1943 poussent à enterrer les lieux de production. La guerre oblige à produire toujours plus, alors même que le nombre d'Allemands appelés au front augmente. La solution passe donc inévitablement par la main-d'œuvre concentrationnaire. Les acteurs - qui sont aussi les profiteurs - sont la SS mais aussi les industriels. Quant aux victimes, ce sont les détenus, de plus en plus nombreux. De nouveaux camps sont ouverts, à Lublin, à Dora, à Riga, Kaunas et Vaivara (Baltique), à Cracovie (Plaszów). Les courbes deviennent exponentielles: 110000 détenus en septembre 1942, 203000 en avril 1943, 524286 en août $1944^{(17)}$. En 1945, plus de 700000 individus, alors encore détenus sous différentes catégories - rescapés en sursis des camps de concentration et d'extermination -, sont évacués pour éviter leur libération par les Alliés, tentent de se maintenir en vie lors des marches de la mort. Cet épisode tragique ${ }^{(18)}$ est l'une des plus terrifiantes illustrations de ce que fut le régime nazi dans sa négation catégorique de l'homme.

\section{Natzweiler: une histoire originale}

Dans cette histoire générale du système concentrationnaire, Natzweiler occupe à maints égards une place particulière et qui demeure paradoxalement, nous le disions déjà, encore très peu connue ${ }^{(19)}$.

Sis dans une Alsace annexée de facto au Reich en juin 1940, Natzweiler est le seul KL sur le territoire français actuel. L'intérêt que porte la DEST à la découverte faite par le SS-Standartenführer Heinz Blumberg, en septembre 1940, d'une carrière de granit rose de très belle qualité sur le mont Louise dans les Vosges, près du village de Natzwiller devenu Natzweiler, détermine la décision d'implanter un camp à proximité pour exploiter la pierre. Le cas est identique à celui qui présida à l'implantation des KL de Mauthausen et de Flossenbürg.

16 À Neckarelz et Mannheim, kommandos de Natzweiler, les détenus occupent une école vidée de ses élèves.

17 M. Broszat, « Nationalsozialistische Konzentrationslager» (note 7), p. 131 sq.

18 On peut renvoyer à Daniel Blatman, Les marches de la mort. La dernière étape du génocide nazi, Paris, Fayard, 2009 et à I. Kershaw, La Fin (note 4), qui donne des analyses éclairantes.

19 R. Steegmann, Le camp de Natzweiler-Struthof (note 11), p. 45-56. 
Le camp doit être aménagé sur un terrain qui appartient à un fermier également propriétaire d'une auberge-hôtel sur le lieu-dit du Struthof. Le terrain choisi est à $750 \mathrm{~m}$ d'altitude sur un versant à forte pente exposé aux vents glacés du nord. Le lieu est bien connu des Strasbourgeois qui, avant la guerre, venaient nombreux s'y promener en été ou profiter de la neige en hiver. Le site offre l'avantage d'être à proximité de la petite ville de Rothau, dans la vallée de la Bruche, placée sur la ligne directe du chemin de fer depuis Strasbourg $(50 \mathrm{~km})$. Hommes et matériel peuvent être assurés pour le fonctionnement du camp qui reste à construire. Avant de s'y installer, les premiers arrivés (539 sont immatriculés en 1941) commencent sa construction. Dans l'attente, ils sont hébergés dans un bâtiment annexe sis en face de l'hôtel. C'est le site de la future chambre à gaz. En février 1942, les détenus sont implantés dans le camp encore en travaux. L'ouverture du block-crématoire, le 14 octobre 1943, marque la fin des constructions. L'hôtel sert alors aux SS.

Le camp est aménagé en terrasses dotées chacune de deux blocks séparés par une place d'appel ${ }^{(20)}$. Au bas du camp, se trouvent le Bunker (prison) et le crématoire. Les dix-sept bâtiments sont entourés de huit miradors et d'une double rangée de barbelés, le tout occupant une superficie d'à peine 1 ha. Natzweiler est donc un petit camp, ce qui n'ôte en rien son caractère terrible, bien au contraire. Géographie, climat et topographie participent à l'anéantissement des hommes entassés dans un espace réduit, sans possibilité de se faire oublier. En 1944, ils sont près de 6000 dans un espace à peine prévu pour la moitié.

La carrière est à environ $800 \mathrm{~m}$, ceinte de barbelés et dotée de treize blocks-ateliers destinés, dès 1942, à la réparation de moteurs d'avion Junkers. Avec les bâtiments de la SS construits de part et d'autre du chemin qui mène au portail d'entrée, la villa du commandant - avec piscine - située à $100 \mathrm{~m}$ du camp, et l'hôtel et ses annexes (chambre à gaz et ateliers SS), l'ensemble du camp couvre une superficie totale de 4,5 ha.

L'histoire du camp s'y écrit avec sa «normalité » et ses spécificités. Le 28 avril 1941, à l'hôtel du Struthof, Hans Hüttig signe sa première ordonnance en tant que nouveau commandant. Il annonce l'ouverture officielle pour le $1^{\text {er }}$ mai, conformément à l'ordonnance de Himmler en date du 14 avril $^{(21)}$. Hüttig est assisté du Schutzhaftlagerführer Josef Kramer venu de Dachau et d'une soixantaine d'officiers, sous-officiers et gardiens, tous SS. Ne manquent alors que les structures et, surtout, les détenus. Les premiers arrivent le 21 mai 1941. Ils sont 150 suivis, le 23 mai, de 150 autres. Tous viennent du KL de Sachsenhausen. Les droits communs et des Asociaux forment l'essentiel de ce premier contingent (65,7\%). Politiques et déserteurs et réfractaires de la Wehrmacht $\left(\mathrm{SAW}{ }^{(22)}\right)$ ne représentent que $34,2 \%$ du total. Leur nombre croit rapidement dès l'arrivée du troisième convoi depuis Sachsenhausen (29 juin) et du premier de Dachau (27 juin). On compte déjà 40,7 \% de "rouges ", mais encore 49,3\% de "verts » et " noirs ». Le premier mort est enregistré dès le 23 mai, déjà pour « insuffisance cardiaque ", terme générique en usage dans les registres de décès des camps, masquant aisément toute autre cause de décès que naturelle. À la fin de l'année 1941, 539 détenus

20 Pour une description plus détaillée, voir R. SteEgmann, Struthof (note 2), p. 302-316.

21 Bundesarchiv Berlin Lichterfelde (BAB), NS4-Na, Kommandantur-Befehl, 28 avril 1941. 
sont immatriculés à Natzweiler, venus de Sachsenhausen, Dachau et Buchenwald. Une autre remarque peut être faite quant à la dénomination des arrivants. L'arrivant est un Zugang (" arrivage ») et non un Zugänger (" nouvel arrivant »). Il est ainsi une pièce rajoutée, un Stück et cesse d'être un homme. Tout est déjà, et aussi, dans la sémantique ${ }^{(23)}$.

Jusqu'en septembre 1942, Natzweiler est encore un Geschlossenes Lager (« camp fermé ») et ne peut recevoir d'autres détenus que ceux déjà internés dans un autre camp. En septembre, il devient un "camp d'affectation »(Einweisungslager): les détenus peuvent lui être directement affectés par les services de police ${ }^{(24)}$. Natzweiler entre dans la «normalité » concentrationnaire et les effectifs croissent tout en restant modestes: 1467 nouvelles immatriculations en 1942, mais 4089 en 1943. Les origines sont multiples ${ }^{(25)}$, les catégories étendues (ainsi les NN) et les transferts sont nombreux car Natzweiler participe dès lors à la rotation des hommes entre les camps. Simple marchandise, ils sont transposables, car utilisables, dans toute autre structure concentrationnaire. À la fin de 1942, Natzweiler reste encore un petit camp. Dirigé par Josef Kramer ${ }^{(26)}$, il ne compte que 921 détenus sur place. Au même moment, Buchenwald en compte près de 84000 et Sachsenhausen ${ }^{(27)}$ 16577. Malgré la faiblesse des effectifs le premier kommando extérieur ouvre à Obernai le 15 décembre.

La «normalisation » vient en 1943. Les immatriculations quadruplent (4089) et le camp est achevé dans sa construction. Toute l'Europe est présente. Polonais et Soviétiques forment $35 \%$ de l'ensemble des détenus. Les Allemands et assimilés régressent (22 \%) alors que les détenus d'Europe occidentale augmentent: Français, Norvégiens, Néerlandais. Les détenus politiques sont devenus largement majoritaires.

Si le camp se "normalise ", au sens que la SS peut donner à ce terme (s'y trouvent ainsi un orchestre, une bibliothèque et même une salle de cinéma), il développe des éléments qui lui sont plus spécifiques. 1943 est aussi le moment de l'irruption de la science nazie dans le camp. Hirt, Bickenbach et Haagen, tous trois professeurs de la Reichsuniversität ouverte à Strasbourg en 1941, se servent de ce lieu discret pour pratiquer leurs sinistres expérimentations médicales. La chambre à gaz est opérationnelle dès le 12 avril 1943. Expérimentations sur le typhus, sur le gaz phosgène et gazages pour la constitution d'une collection anatomique de Juifs sont entre autres pratiqués dans l'enceinte du camp sur les cobayes humains mis à leur disposition par le système concentrationnaire ${ }^{(28)}$.

23 Michel Fabréguet, Mauthausen. Camp de concentration national-socialiste en Autriche rattachée (1938-1945), Paris, Honoré Champion, 1999, p. 103; Joseph Rovan, Contes de Dachau, Paris, Seuil, 1993, p. 61.

24 BAL, Metz Ordner 8, PV. M. Wochner, 5 janvier 1950, f. 33656-33665.

25 Analyse détaillée dans R. SteEgmann, Struthof (note 2), p. 44-223 pour les arrivées, départs et morts.

26 Kramer qui est le Schutzhaftlagerführer depuis 1941, succède à Egon Zill muté en septembre 1942. Zill a lui-même pris la suite de Hüttig à la fin de janvier 1942. Pour les biographies des commandants, voir R. SteEgmann, Struthof (note 2), p. 322-332.

27 Benz/Distel (dir.), Der Ort des Terrors (note 6), vol. 3, p. 312 (Buchenwald) et 29 (Sachsenhausen).

28 Outre les travaux cités de l'auteur, la thèse de doctorat en médecine de R. Tolédano, Les expériences médicales du Professeur Eugen Haagen de la Reichsuniversität Straßburg: faits, contexte et procès d'un médecin national-socialiste, Université de Strasbourg, 2010, donne l'analyse la plus récente. 
L'arrivée, le 15 juin 1943, du premier convoi de détenus NN - ils sont Norvégiens - marque la deuxième évolution spécifique du camp ${ }^{(29)}$. Français, Norvégiens, Belges et Néerlandais sont les victimes du décret Keitel qui, le 12 décembre 1941 et le 16 avril 1942, précise cette nouvelle catégorie destinée à disparaître sans laisser de trace. Destiné à regrouper tous les NN présents dans d'autres camps, Natzweiler n'a pas eu le temps de remplir ce rôle. L'avancée de troupes alliées depuis juin 1944 et la suppression de la catégorie par le décret « Terreur et sabotage » du 30 juillet 1944 l'en empêchent. À cette date, 2443 NN sont passés par le KL-Natzweiler. Tous résistants, ils ont fortement marqué de leur présence l'ensemble des détenus par leur solidarité, leur résistance aux sévices particuliers qu'ils ont eu à subir.

L'année 1943 reste également celle de l'augmentation des exécutions opérées dans ce lieu discret, comme celle des 13 réfractaires de Ballersdorf, le 17 février $1943^{(30)} 1943$ prépare ici aussi l'année qui s'ouvre.

\section{4 : l'entrée dans la production}

1944 est pour Natzweiler celle de l'augmentation du nombre des hommes, de l'extension spatiale des kommandos, mais aussi celle de sa première mort.

L'observation simple des immatriculations nouvelles suffit ici. De janvier au 31 août 1944, 23199 arrivées sont enregistrées. Les mois de juillet (3577) et d'août (environ 9800) représentent à eux seuls $57,7 \%$ du total. Plus que jamais, toute l'Europe est sur place. Polonais et Soviétiques sont les plus nombreux, suivis par les Français. Parmi eux, également des femmes, car le camp est désormais au centre d'une nébuleuse de kommandos devenus ses satellites.

Conformément à la mutation du système concentrationnaire opérée à la suite de la création du SS-WVHA, Himmler ordonne d'augmenter les internements ${ }^{(31)}$ et Glücks intime aux médecins des KL de réduire la mortalité( ${ }^{(32)}$ - aveu implicite de leur rôle joué jusqu'alors! Le résultat reste ici très relatif, car c'est bien l'augmentation de la répression exercée dans toute l'Europe qui maintient et augmente les effectifs.

Après Obernai, le 15 décembre 1942, six kommandos extérieurs sont ouverts en 1943: deux en Moselle - Peltre et Metz -, quatre sur la rive droite du Rhin - Heppenheim, Ellwangen, Iffezheim et Schömberg ${ }^{(33)}$. À ce moment, seul ce dernier est intégré au processus économique, les autres assurant des services pour la SS. En 1944, le nombre des créations de camps annexes se poursuit de part et d'autre du fleuve. Sur les territoires de l'Alsace-Moselle, huit sont ouverts: Sainte-Marie-aux-Mines, UrbèsWesserling, Sennheim, Thil, Hayange, Colmar, Schwindratzheim et Mulhouse. Ils sont plus nombreux sur la rive droite: Cochem Frommern, Neckarelz, Leonberg, Erzingen,

29 Joseph de La Martinière, Le Décret et la Procédure Nacht und Nebel, Paris, FNDIRD, 1989 (2éd.); pour une synthèse sur Natzweiler, R. STEEGMANN, Le camp de Natzweiler (note 11), p. 79-82.

30 R. SteEgmann, Struthof (note 2), p. 57-59.

31 BAB R 58/1027a, 28 mars 1943 et Doc. Nuremberg PS-1063, 17 décembre 1942, Müller aux responsables de la Sipo et du SD.

32 Doc. Nuremberg, Dok-NI-10815, 28 décembre 1942.

33 Benz/Distel (dir.), Der Ort des Terrors (note 6), vol. 6, p. 48-190, pour une monographie des kommandos. 
Geislingen, Francfort-Walldorf et Katzbach, Vaihingen, Dautmergen et Darmstadt. Aux plus petits (Frommern, 120 détenus) s'opposent les plus grands: Neckarelz (2754) et Vaihingen (2 189). Le premier (en cinq mois) et le second (en un mois) totalisent chacun un nombre de détenus présents égal à celui du camp-souche à la fin de sa troisième année d'existence (2800).

L'envolée du nombre implique une modification du fonctionnement du KL et de celle des convois. En 1944, Natzweiler est surtout un sas de passage et de tri avant une affectation dans un camp annexe. De fait, la grande majorité des détenus alors immatriculés par le sigle administratif « KL-Na » ne connaissent pas leur camp-souche. Au 2 septembre 1944, sur 23199 immatriculations depuis janvier, 18151 (dont 2398 femmes juives) sont détenus dans un camp extérieur et on peut estimer à 12000 ceux qui n'y sont jamais passés.

Natzweiler est entré dans la production pour l'économie de guerre. Après les kommandos au service de la SS - à Obernai, par exemple, l'école féminine des auxiliaires SS des transmissions -, les nouveaux, à l'instar de Schömberg (décembre 1943), sont exclusivement destinés à permettre de poursuivre la guerre. Entretien et remise en état d'infrastructures pour le compte de l'armée, ou production pour l'industrie de guerre, "la liste des profiteurs se lit comme un annuaire des entreprises allemandes ${ }^{(34)}$. Toutes les entreprises allemandes, des plus grandes aux plus petites - souvent leurs sous-traitantes -, ont utilisé et usé de la main-d'œuvre des concentrationnaires. Volontaires, suivistes, victimes, leurs cas sont à observer et il reste ici encore de la matière pour des recherches futures. Car seule la ténacité des historiens a permis de faire avancer la lente reconnaissance des faits.

Avant le tournant de septembre 1944, l'exploitation économique des détenus permet d'observer différentes situations dont on donnera par la suite quelques exemples significatifs. On distingue les kommandos affectés au service de l'industrie de guerre, de la Luftwaffe et de la SS comme celui, temporaire, de Hayange qui reçoit 500 femmes juives venues d'Auschwitz (24 ou 28 août). Celui de Mulhouse (30 août) prévu pour la Elsässische Maschinenbau $\mathrm{GmbH}$ (Elmag) est tout aussi éphémère ${ }^{(35)}$. Il y a là deux illustrations parfaites à la fois de la lourdeur et de la rigidité du système administratif des camps que rien ne peut arrêter, sauf l'imprévu: l'évacuation ordonnée de la rive gauche du Rhin. Ils sont prévus, les détenus arrivent, mais repartent avant même d'avoir pu être utilisés sur place. Leur sort se joue par la suite ailleurs, sans que leur travail ne soit perdu.

Darmstadt est un petit kommando. Il compte alors 12 détenus venus le 31 août 1944 de Sachsenhausen: tous des professionnels affectés à la Maschinen-Fabrik Heymann qui produit des pièces pour les V2 et des appareils d'optique. À Francfort-Walldorf, l'Organisation Todt est chargée d'aménager un site pour la construction des avions Messerschmitt: 1700 Juives en majorité hongroises arrivent de Birkenau le 22 août. À Francfort-Katzbach, 200 hommes viennent de Buchenwald à la fin du mois d'août, vite rejoints par d'autres: ils sont affectés aux Adlerwerken $A G$, le quatrième constructeur allemand de camions.

34 Wolfgang Sofsky, L’organisation de la terreur, Paris, Calmann-Lévy, 1995, p. 228.

35 Les femmes de Hayange partent le 2 septembre vers Ravensbrück et les hommes de Mulhouse le 29 septembre vers Dachau avant de prendre le chemin de Flossenbürg. 
Ailleurs, le travail se fait dans des usines souterraines qui se déploient dans une Allemagne devenue la proie des bombardements quotidiens. Au printemps 1944, des "états-majors spéciaux » (Sonderstäbe) sont créés pour répartir le travail des détenus en fonction de l'urgence des besoins. Trois d'entre eux touchent Natzweiler: le Jägerstab pour l'industrie aéronautique; le Geilenbergstab pour le raffinage du pétrole et le Sonderstab-Kammler qui coordonne la création des usines souterraines ${ }^{(36)}$. Vingt installations sont prévues, divisées en deux groupes (A et B) et dotées chacune d'un nom de code avec des dénominations de minéraux (Kaolin,...) ou d'animaux. À Cochem, sur les rives de la Moselle, ouvre le premier kommando enterré de Natzweiler (14 mars 1944). Connu sous le nom de Zeisig («tarin »), les détenus doivent aménager un tunnel pour l'entreprise Bosch de Stuttgart. À Urbès-Wesserling (15 mai 1944), près du col de Bussang dans les Vosges, c'est un tunnel ferroviaire qu'il faut transformer pour une annexe de la Daimler-Benz-Werke $G m b H$ de Colmar et de la Flugmotoren-Werke de Reichshof (projet A10 - Kranisch, "grue »). À Sainte-Marie-aux-Mines, le tunnel sousvosgien est prévu pour la $B M W$ d'Allach. À Leonberg, le travail est fait dans le tunnel routier pour Messerschmitt. Le cas de Neckarelz (projet Goldfish, " poisson rouge ») est bien plus vaste encore et débute en mars 1944. Par la suite, après l'évacuation du camp principal, Neckarelz devient le nouveau centre du complexe concentrationnaire de Natzweiler et d'un réseau de sous-camps. Le projet est destiné à Daimler-Benz et occupe plusieurs dizaines d'entreprises locales ou non ${ }^{(37)}$. Un autre complexe de kommandos formant le Groupe Wüste (" désert ») commence à se mettre en place avec l'ouverture de Schömberg en décembre 1943. Il s'agit ici de parvenir à transformer les schistes pour produire du carburant ${ }^{(38)}$.

La liste des lieux et des entreprises serait longue. La mine de fer désaffectée de Tiercelet à Thil doit être encore mentionnée. Le cas est unique dans l'histoire concentrationnaire puisque localisé en France occupée, de l'autre côté de la frontière de la Lorraine annexée. Choisi pour pallier le bombardement de Peenemünde (17-18 août 1943) afin de poursuivre la construction des V1 et surtout des V2 qui est alors dispersée également à Dora (KL-Buchenwald) et à Ebensee (KL-Mauthausen), le travail est effectué à Thil sous la conduite personnelle du professeur Porsche.

Tous ces sites sont en place au début du mois de septembre quand arrive l'ordre d'évacuer le camp principal. L'avance des Alliés pousse également à l'évacuation des kommandos extérieurs situés sur la rive gauche du Rhin. Il faut à présent y revenir.

\section{Il faut vider la rive gauche du Rhin (septembre/novembre 1944)}

Deux détenus - un Luxembourgeois et un ingénieur soviétique - qui réparent des postes de radio dans l'un des blocks ateliers qui surplombent le camp-souche, s'arrangent pour en garder un toujours en état de marche. Ils peuvent entendre Radio Berlin, Radio Londres, Radio Luxembourg et même, plus difficilement, Radio

36 Sur cette question au développement complexe, voir R. STEEgmann, Le Struthof (note 2), p. $270-274$.

37 Benz/Distel (dir.), Der Ort des Terrors (note 6), vol. 6, pour les monographies des différents kommandos de ce complexe, p. 132-152.

38 Christine Glauning, "Bisingen und das Unternehmen Wüste », in: Benz/Distel (dir.), Der Ort des Terrors (note 6), vol. 6, p. 55-63. 
Moscou $^{(39)}$. Le 6 juin 1944, l'information du débarquement est immédiatement transmise à la résistance intérieure du KL. Naît un fol espoir: "Certains copains croyaient déjà que nous serions libérés sous quelques jours. [Mais] "la guerre n'est pas finie pour autant” " ${ }^{(40)}$. Elle en est pourtant considérablement modifiée. L'avance des Alliés est plus lente qu'on ne l'espérait à Natzweiler où, sur place, les choses se compliquent et le climat s'alourdit progressivement. Les arrivées sont de plus en plus nombreuses car les prisons allemandes en France sont vidées. Le camp est surpeuplé, et la nervosité gagne les gardiens, obligeant les détenus les mieux informés à redoubler de vigilance. Tout s'accumule à la mi-août et indique que quelque chose se prépare.

C'est donc dans une atmosphère très fébrile depuis plus d'un mois qu'est décidée l'évacuation du camp-souche. La tension et la nervosité sont palpables de part et d'autre. Les rares informations qui filtrent encore de l'extérieur trouvent confirmation dans le flot des nouveaux arrivants qui, sortis des prisons de France rajoutent encore à l'entassement dans le camp. La résistance interne est au courant d'un projet d'action menée par les maquis des Vosges, brisé par les troupes allemandes. Une telle masse est bien difficile à gérer pour la faible garnison SS, déjà bien nerveuse. Elle est aussi une cible facile. Les reculs des troupes allemandes à l'ouest et au sud de la France et la libération de Paris portent espoir autant que crainte. Les détenus n'allaient-ils pas servir d'otages? Ni le renforcement de la surveillance, ni les mitrailleuses en batterie pour protéger le camp d'une attaque extérieure toujours crainte, ni les exécutions massives opérées le $1^{\text {er }} / 2$ septembre ${ }^{(41)}$ ne calment les inquiétudes.

L'évacuation ne donne pourtant pas l'impression de la précipitation, alors même qu'elle est, à plus d'un titre, un événement exceptionnel. Il s'agit non seulement d'organiser le premier transfert sur le sol allemand de la totalité des détenus d'un campsouche et de tous ses satellites de la rive gauche du Rhin, mais surtout de n'en perdre aucun. Tout est préparé. Les documents, en grande partie conservés, attestent de la maîtrise de la situation et d'une capacité étonnante d'adaptation de la part d'une administration très cloisonnée. Certes, la lente avancée des Alliés vers les Vosges laisse du temps - Saint-Dié est libéré le 19 novembre 1944 - et évite une panique qui n'aurait pas manqué de se retourner contre les détenus. En mars 1945, la situation sera bien différente.

Nommé à la tête du camp en mai 1944, Fritz Hartjenstein ${ }^{(42)}$ remplace J. Kramer. Sa formation militaire, sa rigueur et sa discipline, son expérience acquise depuis 1940 dans plusieurs KL lui sont utiles pour exécuter l'ordre reçu le $1^{\text {er }}$ septembre, mais déjà prévu. Ses nombreux déplacements en août sont en partie liés à la préparation d'une opération qui doit être minutieusement réglée. Il se rend lui-même à Karlsruhe pour obtenir de la direction de la Reichsbahn la mise à disposition de six trains qui,

39 Roger Leroy, Roger Linet, Max Nevers, La Résistance en enfer, Paris, Messidor, 1991, p. 285.

40 Ibid., p. 302, citant le général Delestaraint.

41 Dans la nuit du $1^{\text {er }}$ au 2 septembre 1944, 107 membres du Réseau Alliance et 35 maquisards des maquis des Vosges sont assassinés et incinérés au crématoire du camp: R. STEEgmann, Struthof (note 2), p. 92-194.

42 R. Steegmann, Struthof (note 2), p. 328-330. 
depuis Rothau, doivent transporter les détenus. Le KL de Dachau est également averti préalablement de l'arrivée de près de 6000 hommes en septembre et du transfert sur place d'une partie de l'administration de Natzweiler (Effektenkammer, Politische Abteilung).

L'importance des effectifs à déplacer et de la surveillance à mettre en place le long des $8 \mathrm{~km}$ qui séparent le camp de la gare de Rothau, obligent à organiser plusieurs convois. Les listes nominatives sont dressées. Les plus valides iront à pied, les malades du Revier en camion. En partant, chacun « doit avoir un couvert, une cuillère et une gamelle [et] un ravitaillement pour une durée de trois jours doit être prévu » ${ }^{(43)}$.

La minutie de l'organisation n'empêche pas l'imprévu de dernière minute. Le premier convoi de 2400 hommes quitte le KL le $1^{\text {er }}$ septembre, vers 22 heures. À mi-chemin, il faut rebrousser chemin: il manque une locomotive! Tout rentre dans l'ordre le lendemain matin. Trois convois quittent Rothau: le premier dans la nuit du samedi 2 au dimanche 3 septembre, les deux autres le lundi 4 . Ils arrivent deux jours plus tard. Le 8 septembre, un télégramme, adressé à 21 h15 par la Kommandantur de Dachau, parvient à celle de Natzweiler ${ }^{(44)}$. Trois convois ont été réceptionnés et les détenus de Natzweiler sont rentrés dans les effectifs de Dachau: 2400 hommes arrivés le 4 septembre sont enregistrés le lendemain; 1990 hommes arrivés le 6 le sont le 7 et enfin, les 1127 derniers, également arrivés le 6, ne sont immatriculés que deux jours plus tard. Le télégramme déplore l'absence de liste précise pour le dernier convoi, empêchant d'identifier 13 décès constatés à l'arrivée du train. Ignorant la décision prise de ne pas attaquer le camp, mesurant mal l'ampleur des opérations lancées contre les maquis, les membres de la résistance interne gardent le secret espoir d'une libération possible pendant la descente à la gare. Mais rien n'est tenté. Le rapport d'effectifs du 30 septembre confirme bien, à la date du 4 , le départ de 5518 détenus ${ }^{(45)}$. Deux autres suivent, le 14 septembre ( 8 hommes) et le 19 (401 hommes) ${ }^{(46)}$. En deux semaines, Dachau a intégré 5926 détenus supplémentaires. Avec le transfert de 108 détenus vers l'éphémère kommando de Schwindratzheim, et le maintien sur place de 16 hommes au-delà du 20 septembre, il est ainsi possible de fixer avec précision la population détenue au camp-souche. Prévu pour un maximum de 3000 hommes, il en compte plus que le double en septembre 1944 (6050) dont 20 \% sont des malades tirés du Revier.

Le croisement des sources - celles du départ de Natzweiler et de l'arrivée à Dachau permet de cerner les nationalités et les catégories. Les Allemands, majoritaires en 1941, ne comptent plus que pour une faible part $(7,2 \%)$ au regard des Français $(43,5 \%)^{(47)}$. Deux détenus sur trois viennent de l'Europe occidentale (63,2\%). Cette supériorité est alors celle du camp-souche, non des kommandos. La présence NN l'explique en grande partie. L'Europe centrale, orientale et balkanique forme le deuxième groupe: Polonais (13,6 \%), Soviétiques (12,2 \%) et Yougoslaves (2,1\%). Dans les kommandos ils sont alors les plus nombreux. L'Europe méridionale ne compte que pour une faible

43 Ordre d'évacuation, in: ibid., p. 160. 
part (19 Grecs et 18 Espagnols) ${ }^{(48)}$. Natzweiler est un camp de Politiques: Schutzhäftlinge $(62,3 \%)$ et $\mathrm{NN}(24,5 \%)$ forment les quatre cinquièmes de la population. Les " verts " $(1,7 \%)$ et les Asociaux (2\%) sont très minoritaires et sans réels pouvoirs depuis l'accession de Willy Behnke ${ }^{(49)}$ au poste de doyen du camp (Lagerälteste). Loin derrière, mais eux bien plus nombreux dans les kommandos, les travailleurs civils ${ }^{(50)}$ forment un deuxième groupe $(7 \%)$. Tous les autres groupes ne sont que faiblement représentés: SAW (0,05\%), homosexuels (0,05\%), Tziganes $(0,2 \%)$ et Bibelforscher (0,05\%). Depuis 1941, la rotation des hommes a été importante (36 sont arrivés en 1942, 634 en 1943). Seuls douze sont sur place depuis 1941, dont huit des deux premiers convois de Sachsenhausen. Ils sont Allemands, et occupent des fonctions dans la hiérarchie détenue: deux droits communs, deux Asociaux et quatre Politiques dont W. Behnke. Sa présence dans les grands convois de transferts vers Dachau est significative. Son rôle est reconnu tant par les SS que par ses pairs. À Dachau, il maintient la cohésion du groupe de Natzweiler arrivé en surnombre dans un camp déjà bien rempli. Son expérience des rouages et des hommes lui permet d'intervenir auprès de l'administration de Dachau. Mais sa présence est également révélatrice de la nature de cette évacuation de septembre 1944, que l'on voit encore comme une mise à l'abri temporaire des détenus face à une situation militaire estimée réversible. D’autres éléments vont dans le même sens.

Plus de 400 détenus restent sur place avec Hartjenstein et la Kommandantur. Ils ne sont pas choisis au hasard: Politiques (55\%) et NN (29\%) sont les plus nombreux. Plus du tiers sont germanophones, dont 10 Alsaciens-Mosellans et 83 Luxembourgeois alors que ces derniers n'étaient que 60 dans les transferts vers Dachau. Les qualifications professionnelles sont cohérentes: ils sont pour l'essentiel, ouvriers, fondeurs, métallos, mécaniciens, soudeurs, et serruriers. Les autres occupent des fonctions dans la hiérarchie détenue, et sept sont employés aux cuisines.

Dès le départ des trois grands convois, ils doivent remettre les blocks en état pour recevoir une avant-garde de gradés de la Milice, accompagnés de quelques femmes. La cohabitation avec les détenus - en particulier français - est vite tendue, et oblige au transfert des détenus dans les blocks de la carrière où se poursuit le travail de réparation des moteurs d'avions Junkers. Par l'espace occupé, le nombre des hommes et leur activité, la carrière est alors ravalée au rang d'un kommando, à quelques centaines de mètres du camp principal vide de détenus. Près de 3000 miliciens, venus depuis Nancy, occupent les lieux durant une dizaine de jours, avant de repartir vers l'Allemagne ${ }^{(51)}$. Le détenu politique tchèque Franz Kozlik ( $\left.n^{\circ} 980\right)$ a laissé un témoignage sur ce bref passage des miliciens ${ }^{(52)}$. Kozlik, décrit par d'autres détenus, comme " très proche de

48 Les Italiens identifiés sont comptabilisés avec ceux de l'Europe occidentale. Leur repérage est souvent difficile: ils sont assimilés aux Slovènes et aux Croates.

49 Willy Behnke, détenu communiste allemand, porte à Natzweiler le numéro matricule 6. Arrivé par le premier convoi (21 mai 1941) après un parcours concentrationnaire débuté en 1934, il est libéré en 1945 à Dachau.

50 Ausländische Zivil (Zwangs)Arbeiter, catégorie créée après juin 1941.

51 Pierre Giolotto, Histoire de la Milice, Paris, Perrin, 1997, p. 465 sq; et Henry Rousso, Pétain et la fin de la collaboration, 1944-1945, Bruxelles, Complexe, 1984. Giolotto indique 5000 personnes.

52 François Kozlık, Der Berg des Grauens, Strasbourg, SEDAL, 1945, p. 38 sq. 
Kramer et de Hartjenstein qui lui laissaient certaines libertés » ${ }^{(53)}$, a occupé les fonctions de chef de l'orchestre des détenus et de coiffeur des commandants successifs. Des passages de son récit ne laissent d'ailleurs aucun doute sur cette proximité avec les SS. Souvent repris depuis la guerre, son témoignage est, hélas, unique et ne peut donc être confronté à d'autres. Pendant plusieurs jours et plusieurs nuits, l'arrivée des miliciens est suivie d'un incessant va-et-vient de voitures, d'autobus parisiens et de camions remplis de vivres et d'alcools. Darnand et son état-major s'installent et, alors même que Hartjenstein et son administration sont encore là, le drapeau tricolore est hissé dans le camp. Probablement informés de leur présence par la population locale, les Alliés lancent plusieurs opérations de bombardements aériens. Vers le 21 septembre, les miliciens se remettent en route en direction d'Ulm, près de Sigmaringen ${ }^{(54)}$. Presque simultanément, s'effectue le dernier transfert de détenus vers Dachau (401 hommes).

Fin septembre, la page semble définitivement tournée pour le KL de Natzweiler: les détenus ne reviendront pas. Sur place, ne restent plus qu'une partie de l'administration avec le commandant, une garnison d'une quarantaine de gardiens, et 16 détenus (7 Luxembourgeois, 7 Allemands, 1 Lorrain et 1 Autrichien). Tous sont logés dans l'hôtel du Struthof et dans son annexe. Le 11 novembre, Hartjenstein quitte les lieux pour installer la Kommandantur à l'auberge "À la Carpe »(Zum Karpfen), à Guttenbach, dans la vallée du Neckar. Le 22, les détenus encore présents sont transférés par camion à Neckarelz. Dans la descente du camp, mettant à profit une attaque aérienne, deux Allemands et quatre Luxembourgeois en profitent pour s'évader, alors que deux autres sont blessés.

L'histoire du camp-souche se termine au Struthof, là où elle avait commencé en 1941. Mais celle du KL de Natzweiler s'écrit déjà ailleurs, à partir des seuls camps satellites. Le 25 novembre, les soldats de la $6^{\mathrm{e}}$ Armée américaine entrent dans un camp vide. Entre-temps, les kommandos de la rive gauche du Rhin ont été à leur tour évacués. Les détenus - 2898 en septembre (maximum 3036 ) et 1898 en octobre (maximum 1969) sont envoyés dans les camps satellites sur l'autre rive du fleuve: à Neckarelz, pour une partie de ceux de Wesserling et de Sainte-Marie-aux-Mines, ou Kochendorf pour ceux de Thil, par exemple. Le reste part pour Buchenwald (Thil-Longwy et Cochem), Sachsenhausen (Wesserling) et Ravensbrück (Hayange). C'est à nouveau Dachau qui en absorbe plus de la moitié (2546). Pour le seul mois de septembre, près de 7900 détenus de Natzweiler y sont immatriculés. Tous n'y restent pas. Près d'un tiers est récupéré, parfois déjà au bout de quinze jours, et retrouve un kommando de Natzweiler.

\section{L'histoire se poursuit}

Une nouvelle phase commence, marquée par un KL désormais sans camp-souche ${ }^{(55)}$. Cette situation totalement originale ne signifie pas pour autant une diminution de son activité. Bien au contraire. Après un bref flottement administratif, lié à la dispersion

53 BAL Metz Ordner 4.

54 P. Giolotto, Histoire de la Milice (note 51), p. 472.

55 Outre les travaux de l'auteur, Arno Huth, Das doppelte Ende des K.L. Natzweiler auf beiden Seiten des Rheines, Landeszentrale für politische Bildung Baden-Württemberg, 2013, donne la synthèse des recherches les plus récentes. 
des services entre Dachau et Natzweiler, la cadence est vite retrouvée, et permet de reprendre en main la masse des détenus qui arrivent. Plus du tiers des évacués de septembre sur Dachau retrouvent les kommandos de Natzweiler dans le mois, et ils sont alors à nouveau immatriculés. Ceux des kommandos de la rive gauche du Rhin sont conservés et envoyés dans les satellites de la rive droite, où de nouveaux camps sont ouverts, recevant toujours plus de détenus, surtout ceux évacués des camps de l'est et du nord du Reich, fuyant l'arrivée des Soviétiques. La centralisation administrative est retrouvée en novembre 1944 à Guttenbach et à Binau, deux villages proches de Neckarelz. Plutôt efficiente au départ, elle perd vite en efficacité. Tout devrait fonctionner. Mais, ni le contexte, ni les locaux de l'auberge Zum Karpfe ne le permettent. Pourtant, c'est toujours au KL de Natzweiler que l'on s'adresse et que l'on envoie de nouveaux détenus. Comme si rien n’avait changé.

Ce n'est qu'à partir de février 1945 que l'administration SS, poussée à l'itinérance, ne suit plus le rythme des arrivées. La liste matriculaire cesse d'être complétée le 19 janvier $1945^{(56)}$ : le dernier matricule enregistré est le $44599^{\mathrm{e}}$. Passionnante et débridée, la dernière période ne peut être couverte par une documentation directe. Les lacunes peuvent néanmoins être partiellement surmontées, par un travail en aval, à partir des registres d'arrivées de Dachau et des autres camps encore en fonctionnement en mars et en avril 1945. Les enregistrements s'y poursuivent, notant avec précision les origines des nouveaux détenus. Ils sont partis d'un kommando de Natzweiler, ils y étaient donc arrivés. Une estimation fiable porte à 17345 le nombre des arrivées entre septembre 1944 et mars 1945. Elle n'est que minimale. Et pourtant, dans cette structure désormais totalement éclatée qu'est le KL de Natzweiler, et dans un contexte de plus en plus oppressant, elle n'en a que plus de sens. Car, jamais depuis 1941, les conditions de la survie des détenus n'ont été aussi terribles.

Selon les périodes, de septembre 1944 à mars 1945, 30 à 35 kommandos extérieurs continuent de fonctionner, de croître, de tuer les hommes. Tous sont au service d'une guerre que l'on ne veut pas admettre perdue. La mortalité est alors terrible, réduisant l'espérance de vie des nouveaux arrivants, souvent rescapés de longues et pénibles évacuations des camps de l'Est, dont de nombreux Juifs, à peine à trois mois. Le tout s'achève sur les routes avec les marches de la mort. À cette date, le site de Natzweiler a retrouvé un nouvel usage au service de l'administration française. Il est, jusqu'en 1949, un centre pénitentiaire pour les collaborateurs en attente de jugement. Une histoire qui reste à écrire.

En 2014, c'est cette double fin qui est le thème de l'exposition organisée par le Centre européen du Résistant déporté dans le site de l'ancien camp de Natzweiler, simultanément présentée aussi à Neckarelz et ensuite itinérante dans l'ensemble des lieux ayant connu un kommando extérieur.

56 Le dernier rapport complet des effectifs date du 20 novembre 1944: SIR Arolsen Natzweiler 20, f. 63-64. 


\section{Résumé}

En septembre 1944, le camp de concentration de Natzweiler-Struthof est évacué. La longue histoire de la fin des camps nazis débute pour ne s'achever qu'en mai 1945. Le cas de Natzweiler est remarquable. Ouvert en 1941 en Alsace annexée, de taille modeste, il s'insère dans l'ensemble de histoire concentrationnaire débutée dès 1933. La montée progressive des effectifs, leurs origines, le fonctionnement même du camp s'inscrivent dans la "normalité » concentrationnaire. Natzweiler dirige un réseau de kommandos de part et d'autre du Rhin tout en développant des caractéristiques propres. L'une des plus originales est sans doute celle de son évacuation et de sa double fin. Fermé en novembre 1944 sur son site d'origine, il poursuit son existence sur la rive droite du Rhin où il ne cesse de croître. L'année 1944 marque ce tournant pour un camp qui reste encore à ce jour souvent méconnu.

\section{Zusammenfassung}

Im September 1944 wurde das KZ Natzweiler-Struthof evakuiert. Die lange Geschichte des Endes der NS-Lager sollte erst im Mai 1945 abgeschlossen werden. Das Lager Natzweiler ist ein Sonderfall. Von bescheidenem Umfang, 1941 im annektierten Elsass eröffnet, reihte es sich in die 1933 beginnende Geschichte der Konzentrationslager ein. Die allmähliche Vermehrung der Lagerinsassen und ihre Herkunft sowie die Organisation des KZs entsprachen der Normalität der Lager. Aus Natzweiler aus wurde ein Kommandonetz auf beiden Seiten des Rheins geleitet, während das Lager auch ihm eigene Merkmale entwickelte. Seine Evakuierung und sein doppeltes Ende bilden wohl eines der originellsten. Nach der Schließung der ursprünglichen Stätte bestand es auf der rechten Rheinseite weiter und erweiterte sich ständig. Diese Wende markiert das Jahr 1944. 